Z Herz- Thorax- Gefäßchir 2022 · 36:47-52 https://doi.org/10.1007/s00398-021-00475-6 Angenommen: 5. Oktober 2021 Online publiziert: 20. Dezember 2021 (C) The Author(s), under exclusive licence to Springer Medizin Verlag GmbH, ein Teil von Springer Nature 2021

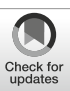

\section{Entwicklung der Herzchirurgie in den letzten 6 Jahrzehnten}

\author{
Von der Hypothermie über die Herz-Lungen-Maschine zur \\ modernen Herzchirurgie
}

Hug Aubin · Artur Lichtenberg · Udo Boeken

Klinik für Herzchirurgie, Universitätsklinikum Düsseldorf, Medizinische Fakultät, Heinrich-Heine-

Universität, Düsseldorf, Deutschland

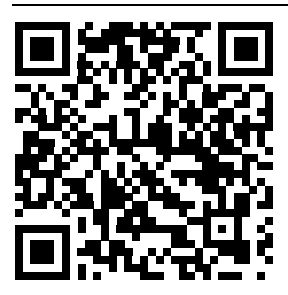

QR-Code scannen \&Beitrag online lesen
Die Entwicklung der Herzchirurgie in Deutschland ist eine Erfolgsgeschichte. Es hat fast 6 Jahrzehnte gedauert, von Ludwig Rehns erster Naht am schlagenden Herzen zur Versorgung einer Stichverletzung 1896 in Frankfurt bis zum Start der Chirurgie am offenen Herzen mit dem ersten erfolgreichen Verschluss eines Vorhofseptumdefekts durch Ernst Derra 1955 in Düsseldorf [1, 2]. Weitere 6 Jahrzehnte später ist die Herzchirurgie eine der lebendigsten und innovativsten chirurgischen Fachdisziplinen in Deutschland, die moderne Chirurgie auf höchstem Niveau betreibt.

\section{Geburt der Herzchirurgie in Deutschland}

Die Herzchirurgie ist eng mit der Geschichte der Chirurgie in Deutschland verwurzelt. Die ersten Pioniertaten im Bereich der Chirurgie am Herzen fanden in der ersten Hälfte des letzten Jahrhunderts maßgeblich im deutschsprachigen Raum statt. Jedoch führten die fatalen historischen Entwicklungen in Deutschland, die im 2. Weltkrieg mündeten, dazu, dass die weiteren Fortschritte im Bereich der Herzchirurgie ab Mitte des letzten Jahrhunderts v. a. aus dem angelsächsischen Raum kamen. Dennoch wurde hierzulande eine ganze Generation von Chirurgen von diesen ersten Pionierleistungen geprägt. Trotz der tiefen Einschnitte, die der 2. Weltkrieg verursacht hatte, blieben somit auch die Protagonisten in Nachkriegsdeutschland immer unmittelbar an den fortschreitenden Entwicklungen der Chirurgie am Herzen beteiligt und verhalfen der Herzchirurgie, eine ei- genständige Fachdisziplin zu werden [3, 4].

Mit Beginn der 1950er-Jahre wurden erstmals klinische Verfahren angewandt, die das sichtkontrollierte Operieren am eröffneten Herzen ermöglichten und somit die operative Korrektur intrakardialer Herzfehler erlaubten [5]. Wegweisend waren die tierexperimentellen Arbeiten zum Kreislaufstillstand in Oberflächenhypothermie von Bigelow in Toronto 1950 [3] sowie die Arbeiten zur extrakorporalen Zirkulation (EKZ) unter Einsatz erster HerzLungen-Maschinen (HLM) von Gibbon in Philadelphia 1953 [6]. Den Widrigkeiten der Nachkriegszeit trotzend, verfolgte auch in Deutschland eine ganze Reihe mutiger Chirurgen diese Entwicklungen und setzte alles daran, die offene Herzchirurgie nachhaltig mitzugestalten [4]. Vor allem zwei Chirurgen stehen stellvertretend für die Geburt der modernen Herzchirurgie in Deutschland: Ernst Derra (1901-1979) und Rudolf Zenker (1903-1984; • Abb. 1).

\section{I) Von Beginn an verfolgten die Protagonisten der Herzchirurgie einen hohen wissenschaftlichen Anspruch}

Lewis und Taufic 1952 in Minneapolis [8] sowie Swan 1953 in Denver [9] hatten angefangen, weiterführende Verfahren zur Behandlung angeborener Herzfehler und Klappenvitien während kurzer Unterbrechungen des Blutkreislaufes unter Hypothermiebedingungen zu entwickeln. In Deutschland verschloss Ernst Derra am 09.02.1955 in Düsseldorf zum ersten Mal einen Vorhofseptumdefekt unter direkter 


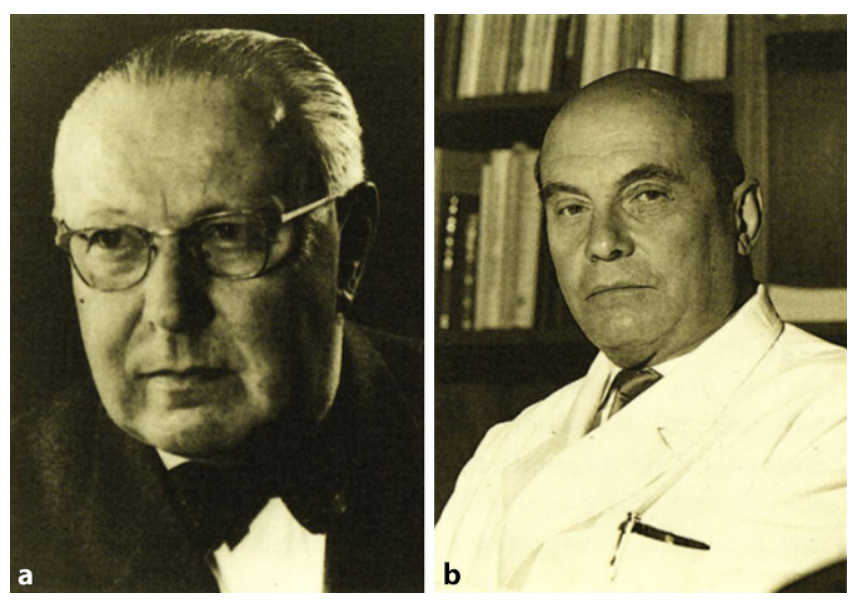

Abb. $1<$ a Ernst Derra (1901-1979); b Rudolf Zenker (1903-1984). (Aus Götze [7])

Sicht im Kreislaufstillstand mithilfe der Oberflächenhypothermie (- Abb. 2, [10]).

Dies war der erste erfolgreiche Eingriff am offenen Herzen im Kreislaufstillstand unter Zuhilfenahme der Oberflächenhypothermie auf dem europäischen Festland und ein Meilenstein für die weitere Entwicklung der Herzchirurgie in Deutschland [10]. Bereits 2 Jahre nach diesem Eingriff wurde 1957 die offene Herzchirurgie mithilfe der Hypothermie in weiteren 8 deutschen Universitätskliniken durchgeführt [4]. Dabei waren die Operationen am offenen Herzen unter Hypothermie v. a. in der Anfangsphase eine immense Herausforderung. Neben den Schwierigkeiten und Tücken der sog. Unterkühlungsanästhesie, die einen nur sehr kurzen Kreislaufstillstand erlaubten, gab es keinerlei Möglichkeit einer genauen präoperativen bildgebenden Untersuchung, sodass häufig sehr komplexe intrakardiale Korrekturen in kurzer Zeit bei unbekannten anatomischen Verhältnissen durchgeführt werden mussten. Beachtenswert ist der schon damals vorhandene wissenschaftliche Anspruch der seinerzeitigen Protagonisten, der Voraussetzung für die Standardisierung der Chirurgie am offenen Herzen und wegweisend für die weitere Entwicklung hin zur modernen Herzchirurgie In Deutschland war. Davon zeugt beispielsweise die weltweit größte klinische Serie von Eingriffen am Herzen mithilfe der Oberflächenhypothermie mit insgesamt 1851 operierten Patienten aus Düsseldorf [12].

Parallel zu den ersten herzchirurgischen Operationen mithilfe der Oberflächenhypothermie wurde auch in Deutschland die
Entwicklung der HLM weitervorangetrieben. Der Einsatz der HLM zur Sicherstellung des Kreislaufs für thoraxchirurgische Eingriffe hatte seine Anfänge in den 1930erJahren in den USA, als Gibbon einen Pumpoxygenator zur Behandlung von Lungenembolien entwickelte. Nach der ersten erfolgreichen klinischen Anwendung der HLM durch Gibbon 1953 in Philadelphia [6] wurde das Prinzip der EKZ für die operative Therapie von Herzfehlern an vielen Kliniken, erst in Nordamerika und schließlich auch in Europa, rasch angenommen und weiterentwickelt [5]. Am 19.02.1958 operierte schließlich Rudolf Zenker in Marburg erstmals in Deutschland erfolgreich einen Patienten mit einem Vorhofseptumdefekt und einer Pulmonalstenose unter Einsatz der EKZ mithilfe einer HLM (• Abb. 3, [13]).

Die Hypothermie verlor nun zugunsten der EKZ an Bedeutung. In rascher Abfolge wurden Patienten schon 1958 in Berlin und München, 1959 in Düsseldorf, Hamburg und Erlangen sowie 1960 in Göttingen, Bonn, Frankfurt, Freiburg, Gießen und Köln erfolgreich am offenen Herzen unter Einsatz von HLM operiert [4]. Die Anwendung der EKZ erlaubte schon bald die sichere Korrektur von angeborenen sowie erworbenen Herzerkrankungen und führte zur flächendeckenden Verbreitung der Herzchirurgie in ganz Deutschland. Die Pionierleistungen von Ernst Derra und Rudolf Zenker, sowie deren Mitstreitern, waren die entscheidenden Impulse, die der modernen Herzchirurgie in Deutschland den Weg ebneten.

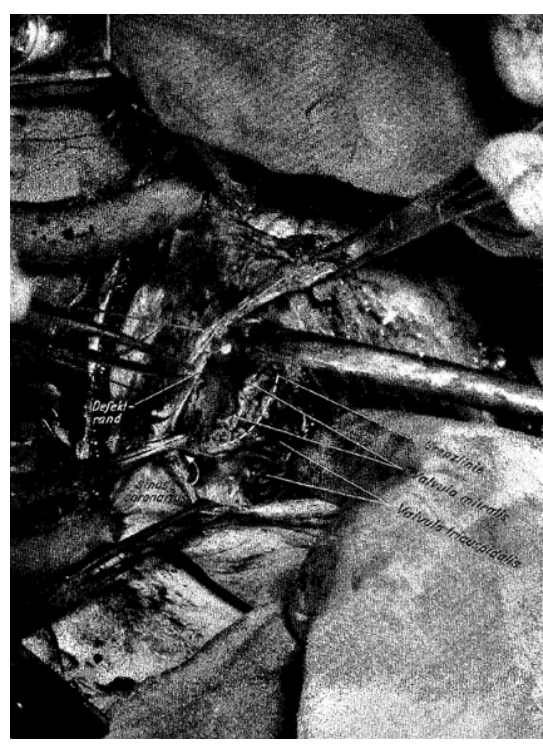

Abb. $2 \Delta$ Verschluss eines Vorhofseptumdefekts durch E. Derra. (Aus Derra [11])

\section{Von den Anfängen bis zur heutigen Zeit}

Gleichzeitig mit der Entwicklung der Chirurgie am offenen Herzen unter Nutzung der EKZ kam es schon bald zu weiteren signifikanten Fortschritten, die für die Weiterentwicklung des herzchirurgischen Operationsspektrums essenziell waren:

- Weiterentwicklung der kardiologischen Diagnostik (Herzkatheter, Echokardiographie, ...),

- Etablierung von geeigneten Intensivstationen,

- Einbindung von nichtärztlichem Personal (z. B. Kardiotechniker an der HLM).

Dies ermöglichte die sichere Durchführung standardisierter Operationsverfahren am offenen Herzen, sodass sich die Operationszahlen im folgenden Jahrzehnt zwischen 1960 und 1970 im noch jungen Fach der Herzchirurgie langsam konsolidierten. Der Schwerpunkt lag anfangs v.a. in der Korrektur angeborener Herzfehler ("congenitals ${ }^{\prime \prime}$ ). Insbesondere die operative Versorgung der sog. Fallot-Tetralogie $[15,16]$ und auch der Transposition der großen Gefäße [17] spielten eine große Rolle. Das Verhältnis von Operationen angeborener im Vergleich zu erworbenen Herzfehlern betrug in den meisten Kliniken zu jener Zeit noch etwa 4:1. Dennoch wurden auch hierzulande die operative Behandlung er- 


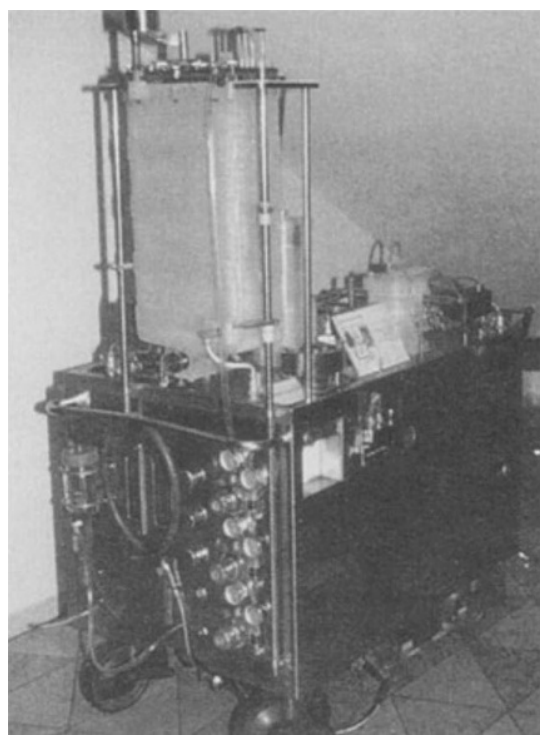

Abb. $3 \Delta$ Herz-Lungen-Maschine der 1. Operation in Deutschland unter Einsatz der extrakorporalen Zirkulation durch R. Zenker am 19.02.1958. (Aus Schmid [14])

worbener Herzfehler mit großen Engagement vorangetrieben und die Grundsteine für die heutigen herzchirurgischen Standardeingriffe gelegt. Beispielsweise wurden, nachdem Harken und Starr 1960/1961 die ersten Herzklappenoperationen durchgeführt hatten, diese Operationsverfahren auch sehr schnell erfolgreich in Deutschland etabliert, mit dem ersten Aortenklappenersatz mit Starr-Edwards-Ball-Prothese 1961 und dem ersten Mitralklappenersatz 1962 in Düsseldorf sowie kurz danach in München [18]. Auch die erste Implantation eines Herzschrittmachers 1961 in Düsseldorf, nur 3 Jahre nach der Erstimplantation von Senning in Stockholm, und die erste Herztransplantation 1969 in München, nur 2 Jahre nach der ersten Herztransplantation durch Christian Barnard in Kapstadt, fallen in dieses Jahrzehnt. Trotz der atemberaubenden Geschwindigkeit, mit der sich die herzchirurgischen Operationsverfahren weiterentwickelten, stiegen die Fallzahlen in Deutschland jedoch nur langsam an: In insgesamt 16 herzchirurgischen Kliniken wurden 1970 lediglich 1975 Operationen durchgeführt [19].

Schon früh hatten die damaligen Protagonisten erkannt, dass der wissenschaftliche Austausch für die Weiterentwicklung der Herzchirurgie unabdingbar war [10]. Bereits 1956 fand eine thoraxchirurgische Arbeitstagung in Bad Schachen am Boden-

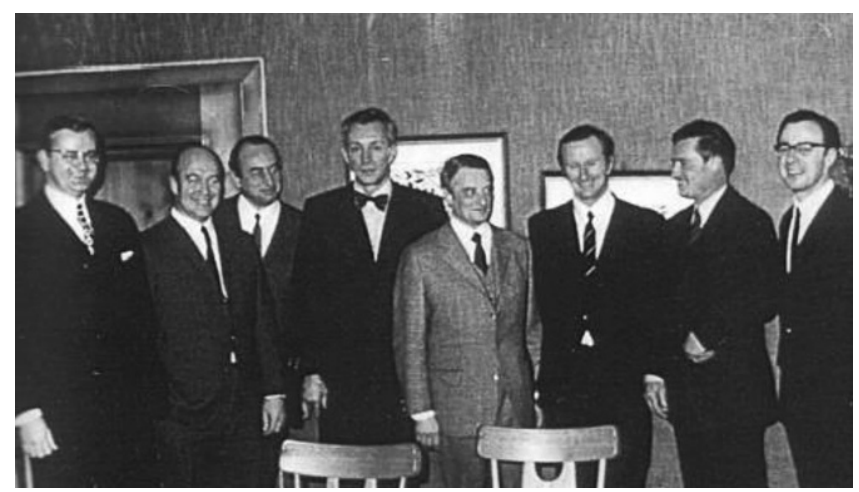

Abb. 4 \ Gründungsmitglieder von links nach rechts: Gall, Hoffmeister, Schlosser, Rodewald, KonCZ, Satter, Stapenhorst und Bircks. Es fehlt Klinner; Borst machte das Bild (09.01.1971). (Aus [20]. Mit freundlicher Genehmigung der DGTHG. Alle Rechte vorbehalten)

see statt. Diese war maßgeblich von Karl Vossschulte (Gießen) und Rudolf Nissen (Basel) angestoßen worden, es beteiligten sich aber auch weitere interessierte Chirurgen, wie z. B. Krauss (Freiburg), Derra (Düsseldorf), Linder (Berlin) und Kunz (Wien). In den kommenden Jahren wurde die Tagung bis 1971 stets in Bad Nauheim durchgeführt und wuchs von Jahr zu Jahr. Zu der Zeit entstanden lange Wartelisten für herzchirurgische Eingriffe in Deutschland, ohne dass die Operationskapazitäten entsprechend zunahmen. Schnell setzte sich bei den damaligen Akteuren die Erkenntnis durch, dass man dem nur durch eine weitere fachspezifische Spezialisierung und der Emanzipation vom Dach der Deutschen Gesellschaft für Chirurgie entgegentreten konnte. So setzte v.a. Josef Koncz 1968 in Bad Nauheim den Anstoß, der am 09.01.1971 in Frankfurt zur Gründung der Deutschen Gesellschaft für Thorax-, Herz- und Gefäßchirurgie (DGTHG) führte. Gründungsmitglieder waren Wolfgang Bircks aus Düsseldorf, Hans Georg Borst aus Hannover, Franz Gall aus Erlangen, Hans Eberhard Hoffmeister aus Tübingen, Georg Rodewald aus Hamburg, Peter Satter aus Essen, Volker Schlosser aus Freiburg, Kurd Stapenhorst aus Homburg/Saar, Werner Klinner aus München sowie Josef Koncz aus Göttingen (0 Abb. 4, [4]).

Die Gründung der DGTHG war neben den ersten Operationen am offenen Herzen von Ernst Derra und Rudolf Zenker, sicherlich der wichtigste Meilenstein, der die Entwicklung der Herzchirurgie in Deutschland nachhaltig vorangetrieben hat.

Das schon früh viel beachtete Publikationsorgan der Herzchirurgie in Deutsch- land war seit 1953 die Zeitschrift Thora$x c h i r u r g i e$ (Thieme-Verlag), deren Name im Juli 1962 in Thoraxchirurgie und vaskuläre Chirurgie erweitert wurde, bevor schließlich 1979 die bis heute gültige Umbenennung in The Thoracic and Cardiovascular Surgeon erfolgte. Durch Borst, der damals die Schriftleitung übernahm, wurde die Internationalisierung der Zeitschrift vorangetrieben. Mit der Änderung der Publikationssprache ins Englische behielt die Herzchirurgie in Deutschland den wissenschaftlichen Anschluss und konnte sich auch weiterhin maßgeblich an der Weiterentwicklung des Fachs beteiligen.

\section{॥) Die Gründung der DGTHG 1971 hat die Entwicklung der Herzchirurgie in Deutschland nachhaltig forciert}

Trotz großen Engagements seitens der DGTHG blieb das Kapazitätsproblem in Bezug auf die herzchirurgische Versorgung in Deutschland jedoch auch in den nächsten Jahrzehnten bestehen. Das stete Anwachsen der Koronarchirurgie, nachdem René Favaloro 1967 in Cleveland erstmalig einen koronaren Venenbypass als Therapie der koronaren Herzerkrankung eingesetzt hatte, führte zum massiven Ansturm von Patienten. Lange Wartezeiten auf eine Bypass-Operation resultierten v.a. in den 1980erJahren in einem regelrechten Auslandstourismus von Koronarpatienten. Denn zwischen 1970 und 1980 war es lediglich zur Entstehung von 5 weiteren herzchirurgischen Kliniken gekommen, sodass 1980 in den nun 21 Zentren insgesamt gerade 


\begin{tabular}{|l|l|}
\hline \multicolumn{2}{|l|}{ Tab. 1 Herzzentren, 1989 } \\
\hline Gründungsjahr & Herzzentren \\
\hline 1958 & Göttingen (J. Koncz) \\
\hline 1965 & Hamburg (G. Rodewald) \\
\hline 1968 & Hannover (H.G. Borst) \\
\hline 1969 & Heidelberg (W. Schmitz) und Freiburg im Breisgau (V. Schlosser) \\
\hline 1970 & Düsseldorf (W. Bircks) und Essen (P. Satter, ab 1973 C. Reidemeister) \\
\hline 1971 & $\begin{array}{l}\text { Tübingen (H. E. Hoffmeister), Gießen (F. W. Hehrlein) und München (LMU; } \\
\text { W. Klinner) }\end{array}$ \\
\hline 1972 & Kiel (A. Bernhard) \\
\hline 1973 & $\begin{array}{l}\text { Frankfurt a. M. (P. Satter), Münster (H. Dittrich) und Homburg/Saar (K. Sta- } \\
\text { penhorst) }\end{array}$ \\
\hline 1975 & Aachen (B. Messmer) \\
\hline 1976 & Köln (H. Dalichau) \\
\hline 1977 & Bonn (P.G. Kirchhoff) \\
\hline 1983 & Würzburg (O. Elert) \\
\hline 1985 & Mainz (H. Oelert) \\
\hline 1986 & Berlin (DHZ; R. Hetzer) \\
\hline 1988 & Ulm (A. Hannekum) \\
\hline Außeruniversitär wurden folgende Einrichtungen gegründet \\
\hline 1974 & München (DHM; F. Sebening) \\
\hline 1978 & Bad Krozingen (M. Schmuziger) \\
\hline 1982 & Kaiserslautern (W. Seybold-Epting) \\
\hline 1983 & Bremen (K. H. Leitz) und Dortmund (St. Johannes, G. Walterbusch) \\
\hline 1984 & Kad Neustadt (R. Hacker), Bad Oeynhausen (R. Körfer), Dortmund (Städtische \\
\hline 1985 & Augsburg (E. Struck) und Fulda (T. Stegmann) \\
\hline 1988 & Bad Nauheim (N. Bleese) \\
\hline 1989 & Buisburg (A. Krian), Rotenburg a.d. F. (H. Oster), Wuppertal (I. Minale) und \\
\hline & \\
\hline
\end{tabular}

mal 10.680 Eingriffe durchgeführt werden konnten. Der damit einhergehende Druck auf die Krankenkassen und die Gesundheitspolitik führte bis 1989 zur Entstehung von weiteren 17 Zentren, sodass insgesamt zum Ende des Jahrzehnts schon über 30.000 Patienten operativ versorgt werden konnten ( $\bullet$ Tab. 1, [21]).

Neben der sich langsam verbessernden Versorgungsituation entwickelte sich auch die Herzchirurgie in Deutschland stetig weiter. Fortschritte im Bereich der Myokardprotektion, die maßgeblich durch Hans Jürgen Bretschneider in Göttingen vorangetrieben wurden, sowie technische Neuerungen im Bereich der HLM ermöglichten neue herzchirurgische Operationsmethoden [10]. Beispielsweise konnten Anfang der 1980er-Jahre die Aortenchirurgie mit der Einführung der "Elephant-trunk"-Methode in Hannover weiter vorangetrieben werden sowie zeitgleich neue Herz- und Lungentransplan- tationsprogramme zunächst in Hannover und München und später in vielen weiteren Zentren etabliert werden. Auch die Erschaffung von mechanischen Unterstützungssystemen und implantierbaren Kunstherzen wurde insbesondere in Berlin maßgeblich vorangetrieben und ihr Einsatz in den Kliniken etabliert. In den folgenden Jahrzehnten wurden zudem minimal-invasive Operationsmethoden im Bereich der Herzchirurgie entwickelt, die auch schnell in Deutschland Verbreitung fanden. Die "Off-pump“-Verfahren im Bereich der Bypass-Chirurgie („off-pump coronary artery bypass", OP(AB), aber auch endoskopische Verfahren im Bereich der Herzklappenchirurgie hielten zunehmend Einzug in die Routineversorgung und wurden von einzelnen Zentren, wie z. B. in Leipzig, entscheidend forciert[19].

Etwa ab dem Jahr 1990 verließ die Herzchirurgie schließlich die geschützte akademische Umgebung der Universitäten, und immer mehr herzchirurgische Einrichtungen wurden deutschlandweit gegründet. Nach einer erneuten Ermittlung des operativen Bedarfs für herzchirurgische Eingriffe und entsprechenden Festlegungen durch die Politik stieg die Zahl der Herzzentren bis zum Jahr 2000 auf ca. 80 an. In diesen Herzzentren wurden bis 2015 jährlich etwa 100.000 herzchirurgische Eingriffe durchgeführt (• Abb. 5).

\section{I) Minimal-invasive herzchir- urgische Operationsmethoden fanden in Deutschland ihre schnelle Verbreitung}

Mit dem neuen Jahrtausend wurden v.a. seitens der interventionellen Kardiologie immer neue, im Vergleich zur Chirurgie minimal-invasivere Therapieverfahren etabliert und in die klinische Routine gedrängt. Hierdurch änderte sich nicht nur das Rollenverständnis zwischen Herzchirurg und Kardiologe, sondern dies erforderte auch eine neue Generation von Herzchirurgen - mit neuer Affinität zu minimal-invasiven und kathetergestützten Verfahren. Die Herzchirurgen kümmern sich seitdem gemeinsam mit den kardiologischen Partnern in "Heart-Teams" um die bestmögliche Therapie der herzkranken Patienten, beteiligen sich aber auch maßgeblich an der Weiterentwicklung und Verbreitung neuer Therapieformen. Die erste perkutane Aortenklappenimplantation erfolgte in Deutschland 2005 in Essen, nachdem der minimal-invasive, transkathetergestützte Ersatz der Aortenklappe (TAVI) 2002 erstmals durch den französischen Kardiologen Alain Cribier in Paris durchgeführt worden war, und breitete sich ab 2007, nach der erlangten CE-Zertifizierung, schnell und mit stetig wachsenden Fallzahlen flächendeckened aus [23]. Schon 2013 wurden in Deutschland mehr TAVI-Prozeduren als chirurgischkonventionelle Aortenklappenersatzoperationen durchgeführt [24]. Vor allem in Deutschland hat die Herzchirurgie diese Entwicklung von Anfang an aktiv mitgestaltet, um sowohl Innovation zu ermöglichen als auch hohe Qualitätsstandards zu gewährleisten. Das ausschlaggebend von der DGTHG vorangetriebene und zusammen mit der Deutschen Gesellschaft für Kardiologie (DGK) initiierte Deutsche Aor- 


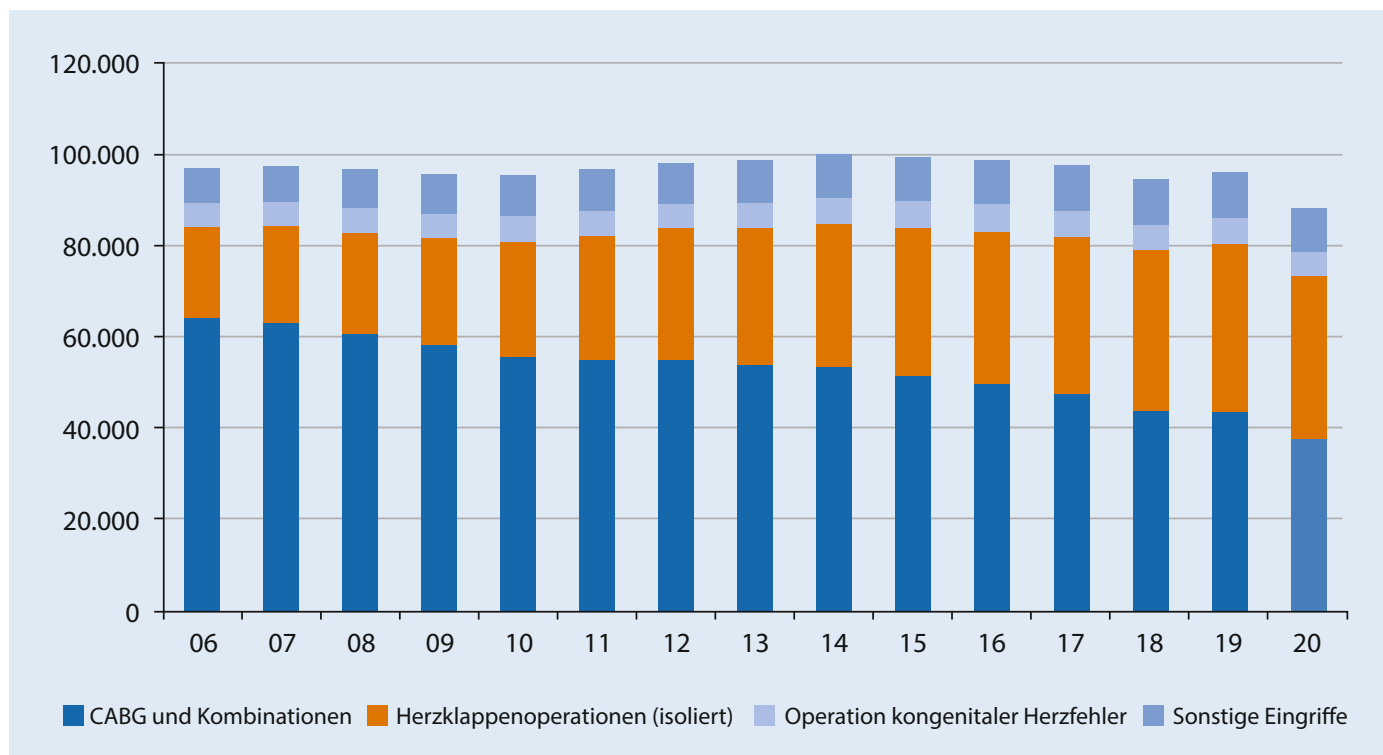

Abb. $5<$ Entwicklung der Operationszahlen in der Herzchirurgie zwischen 2006 und 2020. (Aus DGTHG [22])

tenklappenregister (German Aortic Valve Registry, GARY) ist das weltweit größte wissenschaftliche Register mit Daten von über 140.000 Patienten, auf dessen Basis neue Erkenntnisse bezüglich Nutzen und Sicherheit invasiver Therapien bei Aortenstenose gewonnen werden [25].

\section{I) Die bundesweite Leistungser- fassung der DGTHG sichert höchste Versorgungsstandards}

Seit den Anfängen in den 1950er-Jahren haben sich somit deutschlandweit flächendeckend spezialisierte Herzzentren entwickelt, die heutzutage auf höchstem Niveau das gesamte Spektrum der modernen Herzchirurgie nicht nur abdecken, sondern auch aktiv weiterentwickeln. Die bundesweite Leistungserfassung der DGTHG - einschließlich der Letalitäten - aller beteiligten herzchirurgischen Zentren, die schon 1978 erstmalig etabliert wurde sowie damals und heute wegweisend für eine einheitliche, standortübergreifende Qualitätssicherung ist, sichert höchste Versorgungsstandards. Dabei nimmt die Herzchirurgie hierzulande eine führende Rolle ein, was Qualität und Innovation der chirurgischen Therapie von Herzerkrankungen im 21. Jh. betrifft. Das durch die Fachgesellschaft intensiv geförderte und in der Tradition der Gründerväter der deutschen Herzchirurgie stehende Engagement in experimenteller und klinischer Forschung kommt in der jährlich stattfindenden Jahrestagung und den eigenen Publikationsorganen der DGTHG zum Ausdruck. Mehr noch sind dieses Engagement und die enge Zusammenarbeit mit der Medizinprodukteindustrie in der heutigen Zeit ständiger Motor der Innovation und führen zu einer kontinuierlichen Weiterentwicklung des Faches und der Medizin, auch über den eigenen Fachbereich hinaus [25-27]. Viele herzchirurgische Zentren haben nicht zuletzt eine ganz bedeutende Rolle in der "Coronavirus-disease-2019"(COVID19)-Pandemie gespielt, indem sie existierende Strukturen zur Versorgung von Patienten mit therapierefraktärem Herzund/oder Lungenversagen mithilfe der extrakorporalen Membranoxygenierung (ECMO) genutzt oder diese neu aufgebaut haben, um sich aktiv an der Behandlung von COVID-19-Patienten zu beteiligen [28, 29].

\section{Ausblick}

Die rasante Entwicklung von den ersten Eingriffen am offenen Herzen in Hypothermie zur modernen Herzchirurgie des 21. Jh. in Deutschland wäre ohne die Pioniertaten in den 1950er-Jahren sicherlich nicht möglich gewesen. Die Entschlossenheit der damaligen Protagonisten und ihr Glaube an den medizinischen Fortschritt haben nicht nur die Herzchirurgie, sondern auch die nachkommenden Generationen von Herzchirurgen in Deutschland nach- haltig geprägt. Damals und heute lebt unser Fach von den involvierten Akteuren. Es obliegt deshalb heute uns, nicht nur Patientenversorgung auf höchstem Niveau zu betreiben, sondern auch die Weiterentwicklung der Herzchirurgie wissenschaftlich und praktisch aktiv voranzutreiben, sowie eine neue Generation von Chirurginnen und Chirurgen für die Herzchirurgie zu begeistern und sie zu befähigen, die Herausforderungen der Zukunft zu meistern.

Die Medizin, wie wir sie heute kennen, ist dabei, sich massiv zu verändern. Technologisierung und Digitalisierung schreiten immer weiter voran. Durch den demografischen Wandel, aber auch durch zunehmende Migrationsbewegungen verändern sich die Patientenstrukturen und Krankheitsbilder. Sozioökonomische Zwänge verändern die Rahmenbedingungen. Gesteigerte Patientenautonomie erfordert mehr Transparenz bezüglich Versorgungsqualität und -evidenz. Spezialisierungs- und Subspezialisierungstendenzen nehmen immer weiter zu, genauso wie der Wettbewerb zwischen den einzelnen Fachdisziplinen. Die kommenden Veränderungen werden vielfältig sein und auch die Chirurgie in besondere Weise betreffen. Die Herzchirurgie wird sich unweigerlich verändern; es liegt an uns, heute den Wandel aktiv mitzugestalten und die Weichen für die Zukunft zu stellen, so wie es die Generationen vor uns getan haben. Blicken wir heute auf die letzten 6 Jahrzehnte zurück, können wir 
gemeinsam zuversichtlich in diese Zukunft schauen.

\section{Korrespondenzadresse}

\section{PD Dr. Hug Aubin, MHBA}

Klinik für Herzchirurgie, Universitätsklinikum Düsseldorf, Medizinische Fakultät, HeinrichHeine-Universität

Moorenstr. 5, 40225 Düsseldorf, Deutschland hug.aubin@med.uni-duesseldorf.de

\section{Prof. Dr. Udo Boeken}

Klinik für Herzchirurgie, Universitätsklinikum Düsseldorf, Medizinische Fakultät, HeinrichHeine-Universität

Moorenstr. 5, 40225 Düsseldorf, Deutschland udo.boeken@med.uni-duesseldorf.de

\section{Einhaltung ethischer Richtlinien}

Interessenkonflikt. H. Aubin, A. Lichtenberg und U. Boeken geben an, dass kein Interessenkonflikt besteht.

Für diesen Beitrag wurden von den Autoren keine Studien an Menschen oder Tieren durchgeführt. Für die aufgeführten Studien gelten die jeweils dort angegebenen ethischen Richtlinien.

\section{Literatur}

1. Rehn L (1897) Über Herzwunden und Herznaht. Verh 26Kongr Dtsch Ges Chirl: 72777;Il: 151-165

2. Derra E, Bayer O, Grosse-BrockhoffF (1955) Der Vorhofseptumdefekt und sein operativer Verschluß unterSicht des Auges in Unterkühlungsanästhesie. Dtsch Med Wochenschr 80(36):1277-1281

3. Bigelow WG, Callaghan JC, Hopps JA (1950) General hypothermia for experimental cardiac surgery. Ann Surg 132:531-539

4. Bircks W (2002) "Geschichte der Herzchirurgie in Deutschland" unter Berücksichtigung ihrer Beziehung zu der Deutschen Gesellschaft für Kardiologie - Herz- und Kreislaufforschung. In: von Lüderitz B, Arnold G (Hrsg) 75 Jahre Deutsche Gesellschaft für Kardiologie - Herz- und Kreislaufforschung., Heidelberg

5. Meisner H (2014) Milestones in surgery: 60 years of open heart surgery. Thorac Cardiovasc Surg 62(8):645-650

6. Gibbon HJ (1954) Apllication of a mechanical heart and lung apparatus to cardiac surgery. Minn Med 37:171-180

7. Götze H (1994) Zweiter Abschnitt. In: Der SpringerVerlag. Springer, Berlin, Heidelberg https://doi. org/10.1007/978-3-540-92889-8_2

8. Lewis FJ, Taufic M (1953) Closure of atrial septal defects with aid of hypothermioa; experimental accomplishments and the report of one successful case. Surgery $32: 52$

9. Swan H, Zeavin I, Blount SG, Virtue RW (1953) Surgery by direct visiosn in the open heart during hypothermia. JAMA 153:1081

10. Leitz KH (2010) Geschichte der Herzchirurgie in Deutschland. In: von Ziemer G, Haverich A (Hrsg) Herzchirurgie-Die Eingriffe am Herzen und an den herznahen Gefäßen. , Berlin, Heidelberg
11. DerraE (1958) Die offene Operation des Vorhofseptumdefektes und der valvulären Pulmonalstenose mittels Hypothermie. Arch Klin Chir 289:203-232. https://doi.org/10.1007/BF02432549

12. Schulte HD (2001) "Looking into the past only makes sense if it serves the future."-A reflection on the life and legacy of Ernst Derra on his 100th birthday. Thorac Cardiovasc Surg 49(4):251-253

13. Zenker R, Heberer G, Gehl H et al (1958) Zur Aufrechterhaltung der Organfunktionen und des Stoffwechsels im extrakorporalen Kreislauf. Langenbecks Arch Klin Chir 289:294-302

14. Schmid C, Schmitto JD, Scheld HH (2003) Herztransplantation in Deutschland. Steinkopff, Heidelberg https://doi.org/10.1007/978-3-64257386-6

15. Leitz KH(2006) Videoclip. Aus der ReiheZeitzeugen berichtet Prof. Dr. W. Bircks. DGTHG, Berlin

16. Klinner W, Borst HG, Sebening F et al (1968) Forum Cardiologicum 11. Chirurgie am offenen Herzen. Boehringer, Mannheim

17. Tjindra C (2004) Josef Koncz, sein Leben und Werk Dissertation, Medizinische Hochschule Hannover

18. Schulte HD (2001) A reflection on the life and legacy of Ernst Derra on his 100. birthday. Thorac Cardiovasc Surg 49:251-253

19. Borst HG, Mohr FW (2001) The history of coronary artery surgery - a brief review. Thorac Cardiovasc Surg 49:195-198

20. DGTHG (2021) Gründung der DGTHG. https:// www.dgthg.de/de/Gruendung. Zugegriffen: 23. Nov. 2021

21. Bruckenberger E (1990) Ausbau der herzchirurgischen Operationskapazität in der Bundesrepublik Deutschland. Thorac Cardiovasc Surg 38:262-265

22. DGTHG (2020) DGTHG-Leistungsstatistik 2020. https://www.dgthg.de/sites/default/files/ Grafiken-DGTHG-Leistungsstatistik\%202020_ free-access_0.pdf.Zugegriffen:22. Nov. 2020

23. SackS, Naber C, Kahlert Petal (2005) Die perkutane Herzklappenimplantationin Aortenposition. Herz 30:433-437. https://doi.org/10.1007/s00059005-2726-1

24. Beckmann A, Funkat AK, Lewandowski J, Frie $M$, Ernst M, Hekmat K, Schiller W, Gummert JF, Cremer JT (2015) Cardiac surgery in Germany during 2014: a report on behalf of the German Society for Thoracic and Cardiovascular Surgery. Thorac Cardiovasc Surg 63(04):258-269

25. Beckmann A, Hamm C, Figulla HR, Cremer J, Kuck KH, Lange R, Zahn R, Sack S, Schuler GC, Walther T, Beyersdorf F (2012) The German Aortic Valve Registry (GARY): a nationwide registry for patients undergoing invasive therapy for severe aortic valve stenosis. Thorac Cardiovasc Surg 60(05):319-325

26. Heinemann MK, Tolksdorf M (2014) 60 and counting: the history of "The Thoracic and Cardiovascular Surgeon". Thorac Cardiovasc Surg 62(8):651-655. https://doi.org/10.1055/s-00341384665

27. Aubin H, Boeken U, Lichtenberg A (2015) Herzchirurgie im Wandel der Zeit: Von den ersten eingriffen am offenen Herzen in Hypothermie bis zur modernen Herzchirugie in Deutschland. ZHerz-Thorax-Gefäßchir 29:68-73

28. Aubin H,PetrovG, DalyanogluH,SaeedD, Akhyari $P$ Paprotny G, Richter M, Westenfeld R, Schelzig H, Kelm M, Kindgen-Milles D (2016) A suprainstitutional network for remote extracorporeal life support: a retrospective cohort study. JACC Heart Fail 4(9):698-708
29. Lorusso R, Combes A, Coco VL, De Piero ME, BelohlavekJ(2021)ECMO for COVID-19 patients in Europe and Israel. Intensive Care Med 47(3):344-348 